\title{
A Review on Fog Removal with Its Techniques and Types
}

\author{
Hye-jin $\mathrm{Kim}^{1}$, Suman Tyagi ${ }^{2}$ and Nirupma Tiwari ${ }^{3}$ \\ ${ }^{1}$ Business Administration Research Institute, Sungshin Women's \\ University, 2, Bomun-ro 34da-gil, Seongbuk-gu, Seoul, Korea \\ ${ }^{2}$ Mtech Research Scholars Dept. of CSE/IT, Shri Ram College of Engineering and \\ Management, Banmore, India \\ ${ }^{3}$ Assistant Professor Dept. of CSE/IT, Shri Ram College of Engineering and \\ Management, Banmore, India \\ 1hyejinaa@daum.net, ${ }^{2}$ Sumantyagi30@gmail.com, ${ }^{3}$ Girishniru@gmail.com
}

\begin{abstract}
Nowadays, Images play essential roles in the real world, images define not only the changes in the environment and also use for traffic analysis. But images are captured in an open environment due to the bed whether or atmosphere images are not too good. This paper review the different haze removal processes to eliminate the haze in the captured hazy images to recover better and improved quality haze-free images.
\end{abstract}

Keywords: Image processing, Fog removal, Quality enhancement, Fog removal method

\section{Introduction}

Images of outdoor scenes are degraded by the presence of different particles and the water droplets in the atmosphere. Haze, fog, smoke are such atmospheric phenomena due to atmospheric absorption and scattering. While capturing a scene in the camera in a bad weather condition the irradiance received by the camera from the scene point is attenuated along the line of sight. The incoming light flux is blended with the light from all other directions called the airlight. The amount of scattering depends on the distance of the scene points from the camera; the degradation is variant in nature. Due to this the there is a resultant decay in the color and the contrast of the captured degraded image. [1][2]The target of haze removal is to improve the reflected light (i.e., the scene colors) from the mixed light. The constancy and strength of the visual system can improve by the usage of effective haze removal of the image. There are many methods available to remove haze from an image like polarization, independent component analysis; dark channel prior, etc. [3]

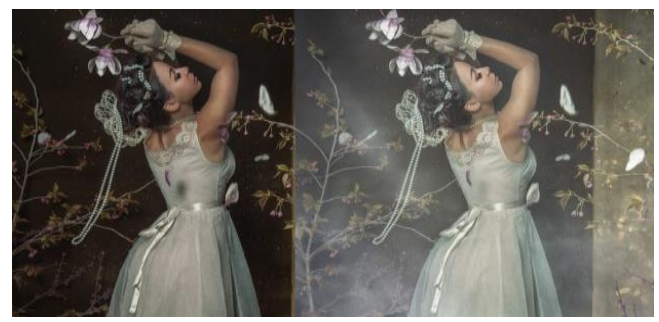

Figure 1.

Article history:

Received (January 29, 2015), Review Result (March 5, 2015), Accepted (April 30, 2015) 
Haze removal is an extreme errand because fog relies on obscure scene depth information. Fog impact is the capacity of separation in the middle of camera and object. Henceforth evacuation of fog requires the estimation of an air light guide or profundity map. The present haze removal strategy can be isolated into two classifications: image enhancement and image restoration. Image enhancement does not include the reasons for fog degrading image quality. This method can improve the contrast of haze images but loses some of the image information. Image restoration firstly studies the physical process of image imaging in foggy weather. After observing that degradation model of fog image will be established. At last, the degradation process is inverted to generate the fog-free image without the degradation. So, the quality of degraded images could be improved.

Different types of noise affect the image. Accordingly, an image enhancement process for improving the quality of an image is needed then. After improving the quality of an image again restore that image. [4]

At present technology for fog removal are of two types-

- Fog correction

- Fog removal

Effect of fog: The effect of fog mainly is caused by two scatterings [1],[2] phenomenon-

1. Attenuation

2. Air-light

\section{Types of fog}

Fog is defined as a cloud with its base at or near the ground. Depending upon the conditions, the ground may become shrouded in radiation fog, advection fog, or up-slope fog.

- Radiation fog: It occurs at night and requires clear skies and fairly high relative humidity. If the air is calm, the fog is patchy.

- Steam Fog: Steam fog Named for the fact that it looks like steam rising from the water is also known as sea smoke, Arctic sea smoke, or evaporation fog.

- Ice-Edge Fog: Ice-edge fog is a band of fog that forms near the edge of sea ice

- Advection Fog: If cooling is sufficient, a blanket of fog called an advection fog will result.

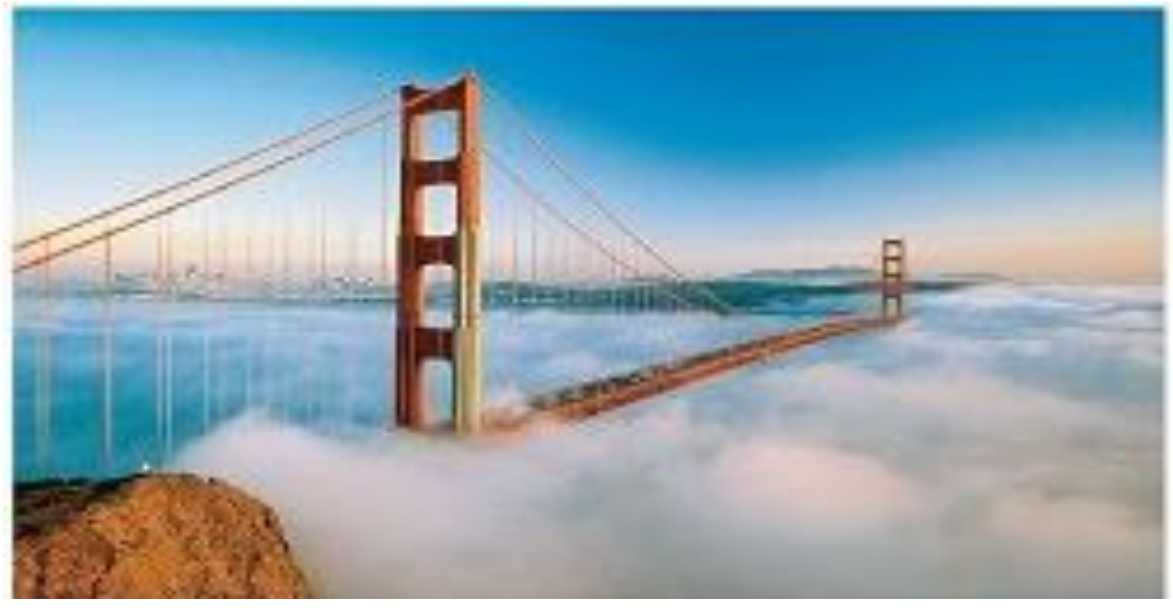

Figure 2. Advection fog 
- Precipitation Fog: As its name suggests, this type of fog is formed by precipitation. It is also known as a frontal fog because it often occurs ahead of warm fronts and behind cold fronts. If the rain moves away, so does the fog.

\section{Image enchantment technique}

Visibility Restoration Technique: for removing haze, fog from the image various techniques are used.[5] typical techniques of image restoration to the haze are:

- Dark Channel Prior: Characterize truncations and acronyms the first time when they are utilized as a part of the content, even after they have been characterized in theory. Shortened forms, for example, IEEE and SI don't need to be characterized. Try not to utilize shortened forms in the title or heads unless they are unavoidable. The dark channel prior (Wang, Yan, et al, 2010) is used for the estimation of atmospheric light in the dazed image to get a more realistic result. This method is mostly used for non-sky patches; one color channel has very low intensity at a few pixels. The low intensity in the dark channel is predominant because of three components:

- Colorful items or surfaces

- Shadows (shadows of cars, buildings, etc.)

- Dark items or surfaces (dark tree trunk, stone)

As the outdoor images are usually full of shadows the dark channels of images will be dark. Due to fog (airlight), a foggy image is brighter than its image without fog. So we can say dark channels of the foggy image will have higher intensity in the region with higher fog along these lines, outwardly the force of the dim channel is an unpleasant estimation of the thickness of the fog. In dim channel earlier we utilize pre and post handling ventures for getting great results. In post preparing steps we use soft matting or trilateral filtering etc. Let $\mathrm{J}(\mathrm{x})$ be the input image, $\mathrm{I}(\mathrm{x})$ is the hazy image, $\mathrm{t}(\mathrm{x})$ is the transmission of the medium. The attenuation of the image due to haze can be expressed as:

$$
\mathrm{I}_{\mathrm{att}}=\mathrm{J}(\mathrm{x}) \mathrm{t}(\mathrm{x})
$$

The influence of fog is Air light effect and it is shown as:

$$
\mathrm{I}_{\text {airlight }}(\mathrm{x})=\mathrm{A}(1-\mathrm{t}(\mathrm{x}))
$$

Dark channel for a random image $\mathrm{J}$, shown as $\mathrm{J}$ dark is defined as:

$$
f^{d a r k}(x)=\frac{\min }{y \in \Lambda(x)}\left(\min j^{C}(y)\right)
$$

In this $\mathrm{J}^{\mathrm{c}}$ is the color image comprising of RGB components, $\Omega(\mathrm{x})$ depicts a local patch which has its origin at $\mathrm{x}$. The low intensity of dark channels is because of shadows in images, color objects, and dark objects in images. After dark channel prior, we need to estimate transmission $\mathrm{t}(\mathrm{x})$ for proceeding further with the solution. After estimating the transmission map depth map is generated. Assume Atmospheric light $\mathrm{A}$ is also known. 

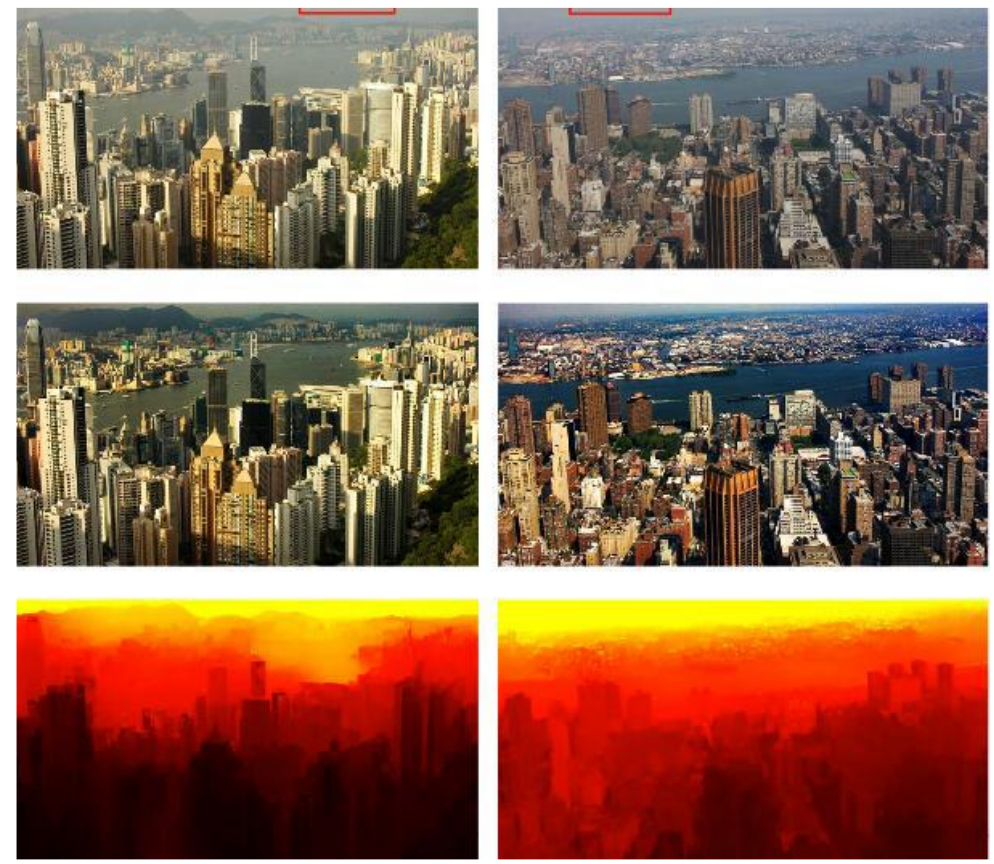

Figure 3. Haze removal results. top: input haze images. middle: restored haze-free images. bottom: depth maps

CLAHE Contrast limited adaptive histogram equalization short form is CLAHE (Xu, Zhiyuan, et al, 2009). Contrast Limited Adaptive Histogram Equalization (CLAHE) is used for the enhancement of low contrast images. This method does not need any predicted weather information for the processing of the fogged image. Firstly, the image captured by the camera in the foggy condition is converted from RGB (red, green, and blue) color space is converted to HSV (hue, saturation, and value) color space. The images are converted because the human senses color similarly to HSV represent colors.

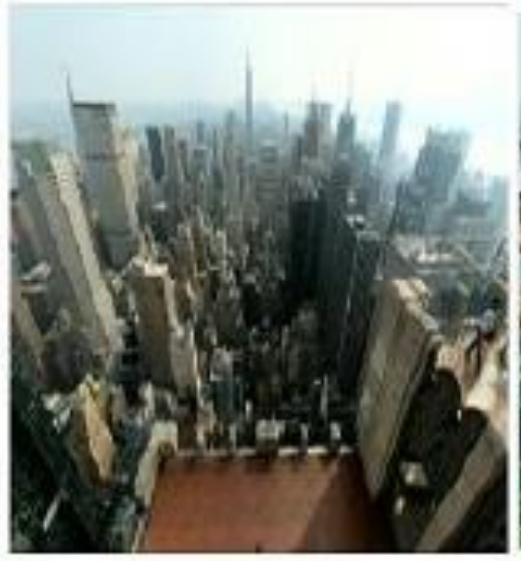

(a)

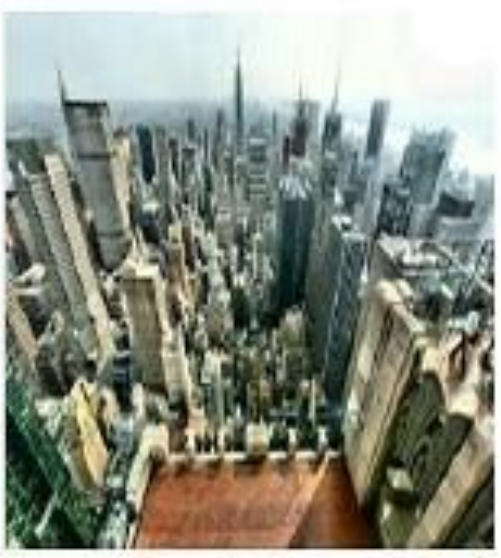

(b)

Figure 4. Shows (a) input image (b) output image 
Secondly, the value component is processed by CLAHE without affecting hue and saturation. This technique use histogram equalization to a contextual region. The original histogram is cropped and the cropped pixels are redistributed to each gray level. In this, each pixel value is reduced to the maxima of user-selectable. Finally, the image processing in HSV color space is converted back to RGB color space. Figure (3) illustrates the results of the improved image using the CLAHE technique.

- Bilateral Filtering: Bilateral filtering smooth's images and also preserves edges, with the nonlinear combination of nearby image values. Bilateral is non-iterative, local, and simple. Gray levels or colors are combined by the bilateral filter based on both their geometric closeness and their photometric similarity and prefer close values to distant values in both domain and range. Bilateral filter smooth edges towards piecewise constant solutions. A bilateral filter does not provide stronger noise reduction. Figure (4) illustrates the processing of foggy images and the establishment of them into the original image by using a bilateral filter.
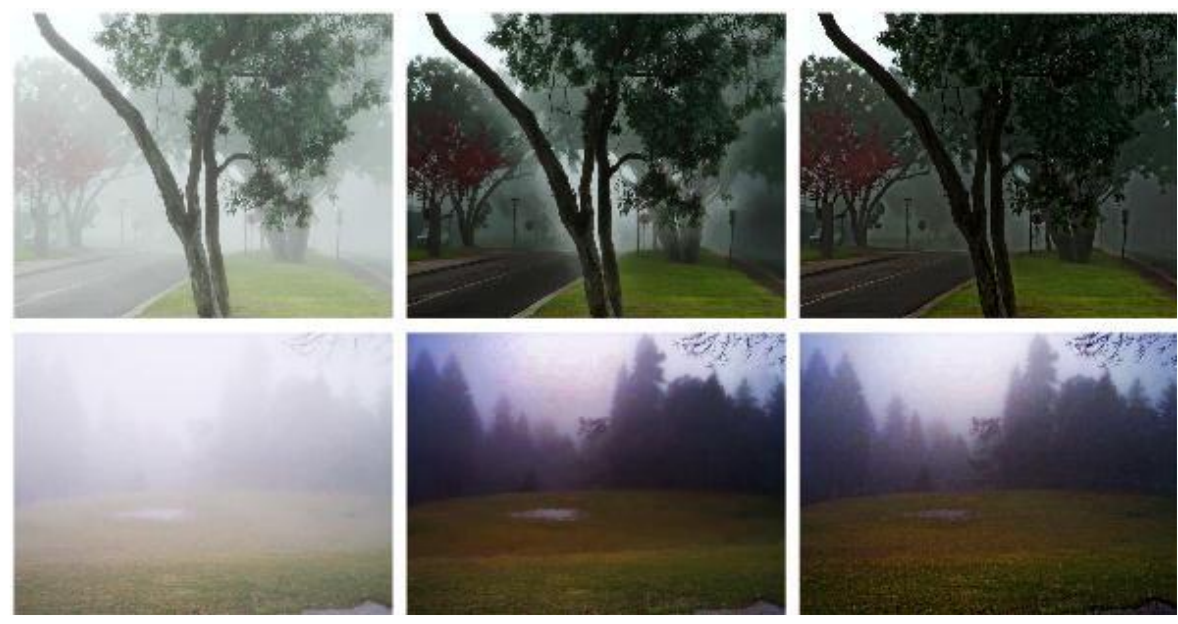

Figure 5. (a) Original foggy image (b) defogged image (c) bilateral defogged image

MIX - CLAHE: Hitam et al. (2013) presented a method to enhance underwater images using a blend Contrast Limited Adaptive Histogram Equalization. The upgrade system adequately enhances the permeability of submerged images and creates the least MSE and the most astounding PSNR values. Along these lines, it has shown that the mix-CLAHE based method is promising for classifying coral reefs especially when visual cues are visible 


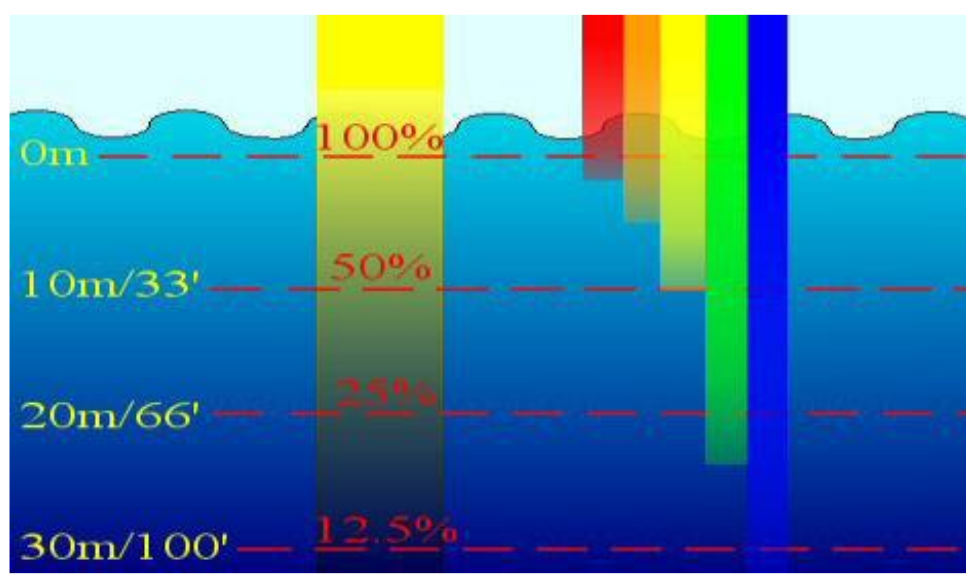

Figure 6. Absorption of light by water

[Figure 5] shows an outline of the ingestion of light by water. For each 10m increment inside and out the brilliance of daylight will drop significantly. Almost all red light is passed by half from the surface yet blue proceeds to awesome profundity. That is the reason most submerged images are overwhelmed by blue-green hues. CLAHE-Mix first standardizes the consequence of CLAHE-RGB. Figure (6) outlines the consequences of the CLAHE procedure working on RGB and HSV shading models and the aftereffect of Mix-CLAHE working on Image. As can be seen from the figure, when CLAHE worked on the RGB shading model, it ruins the human feeling of shading. A more intelligent methodology is to spread the shading values consistently, leaving the hues themselves (e.g., tints) unaltered. The outcome from CLAHE-HSV demonstrates that the general shading is more sensible than CLAHE-RGB. On the other hand, the general image is much brighter and looks unnatural to the image. Also, the unavoidable upgrade of clamor in smooth areas is distinguished. To decrease the undesired relics and additionally shine in CLAHE RGB and CLAHE HSV images we present another system that blends the consequences of CLAHE-RGB and CLAHE-HSV. The strategy is called CLAHE-Mix. The point is to upgrade the image contrast and in the meantime safeguard the normal look of the underwater image.

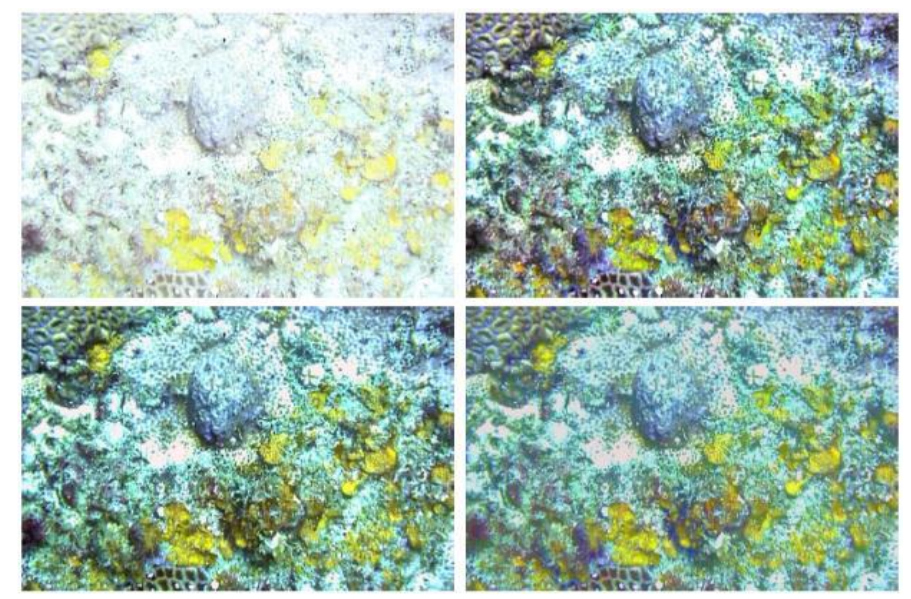

Figure 7. Comparison of CLAHE methods on B1. upper left: original underwater image. upper right:

CLAHE-RGB image. bottom left: CLAHE-HSV image. bottom right: CLAHE-Mix image. 
- Trilateral Filtering: This filtering (Cheng, F.C et al, 2012) smooths images without influencing edges, using a non-linear combination of nearby image values. This filter replaces each pixel with weighted averages of its neighbor's pixel. The weight allotted to each neighbor pixel decreases with both the distance in the image plane and the distance on the intensity axis. This filter helps us to get results faster as compared to others. While using a trilateral filter we use pre-processing and post-processing steps for better results. Histogram stretching is used as post-processing and histogram equalization as a preprocessing

Table 1. Comparison between different image enhancement techniques

\begin{tabular}{|c|c|c|}
\hline S .No. & CLAHE & Mix-CLAHE \\
\hline 1 & $\begin{array}{c}\text { CLAHE stands for Contrast limited } \\
\text { adaptive histogram equalization }\end{array}$ & $\begin{array}{c}\text { Mix-CLAHE stands for Mixture contrast limited } \\
\text { adaptive histogram equalization }\end{array}$ \\
\hline 2 & $\begin{array}{c}\text { CLAHE operates separately on RGB } \\
\text { and HSV color models }\end{array}$ & $\begin{array}{c}\text { Mix-CLAHE mixes the results of CLAHE- RGB } \\
\text { and CLAHE-HSV color models. }\end{array}$ \\
\hline 3 & $\begin{array}{c}\text { The overall image is much brighter and } \\
\text { looks unnatural to the underwater } \\
\text { image. Moreover, the unavoidable } \\
\text { enhancement of noise in smooth } \\
\text { regions is identified. This is the result } \\
\text { of the CLAHE technique operating on } \\
\text { RGB and HSV color models. }\end{array}$ & $\begin{array}{c}\text { The result of mix-CLAHE is the enhancement of } \\
\text { image contrast and at the same time preserves } \\
\text { the natural look of the underwater image. There } \\
\text { is no enhancement of noise in smooth regions is } \\
\text { identified. }\end{array}$ \\
\hline 4 & $\begin{array}{c}\text { CLAHE has a low Peak signal-to-noise } \\
\text { ratio. }\end{array}$ & $\begin{array}{c}\text { Mix-CLAHE has a high Peak signal to noise } \\
\text { ratio }\end{array}$ \\
\hline 5 & CLAHE has a high Mean square error. & Mix-CLAHE has a low Mean square error \\
\hline
\end{tabular}

Both steps help to increase the contrast of the image before and after usage of the trilateral filter. This algorithm is independent of the density of fog so can also be applied to the images taken in dense fog. Table (1) shows a Comparison between different image enhancement techniques such as Clahe and Mix-Clahe.

\begin{tabular}{|c|c|c|}
\hline S. No. & Bilateral Filter & Trilateral filter \\
\hline 1 & $\begin{array}{c}\text { Bilateral filtering smooths images while } \\
\text { preserving edges, using a nonlinear } \\
\text { combination of nearby image values }\end{array}$ & $\begin{array}{c}\text { This filtering smooth's images without } \\
\text { influencing edges, using a non-linear } \\
\text { combination of nearby image values. }\end{array}$ \\
\hline 2 & $\begin{array}{c}\text { Bilateral Filtering is non-iterative, local, } \\
\text { and simple }\end{array}$ & $\begin{array}{c}\text { Trilateral filtering requires more iteration in the } \\
\text { filtering process. }\end{array}$ \\
\hline 3 & $\begin{array}{c}\text { Bilateral filter smooth edges towards } \\
\text { piecewise constant solutions. }\end{array}$ & $\begin{array}{c}\text { Trilateral filter smooth edges towards a sharply } \\
\text { bounded piecewise linear approximation. }\end{array}$ \\
\hline 4 & $\begin{array}{c}\text { A bilateral filter does not provide } \\
\text { stronger noise reduction. }\end{array}$ & $\begin{array}{c}\text { The trilateral filter provides stronger noise } \\
\text { reduction }\end{array}$ \\
\hline
\end{tabular}




\section{Literature review}

Pucheng Zhou et.al [6], this paper present, to enhance the permeability with just a single hazy image, a sort of haze removal algorithm calculation is proposed. Firstly, the crude environmental transmission guide is assessed by utilization of the dark channel prior. And afterward, the Fields of Experts model is received to alter the crude air transmission map. At long last, the scene albedo is restored given the air diffusing model. Investigates an assortment of open-air foggy images to check the achievability and legitimacy of our algorithm. The Fields of Experts model is embraced to get the priors from the degraded image, to revise the environment transmission map. At last, the scene albedo is restored taking into account the environment disseminating model. Analyses demonstrate that the algorithm can be powerful recuperation of the image scene information.

Hiroshi Kawarabuki et. al[7], this paper, we propose a technique for perceiving the level of snowfall consequently even though the greater part of the foundations are secured with snow and the perceivability is low by fog. When it snows vigorously, haze regularly happens all the while. In addition, falling snow grains have a low difference to the background covered with white snow. Keeping in mind the end goal to manage these circumstances, the proposed technique makes an info image clear by fog evacuation. We propose the novel fog removal method which can be connected to the typical scene as well as the heavy snowy scene. This strategy can evacuate the impact of fog without relying upon the evaluation of perceivability because the level of fog evacuation is changed dynamically. The level of snowfall is assessed from the number of falling snow grains, which are removed from the distinction between the present defogged image and the foundation image made by the three-dimensional median.

Shota Furukawa et.al [8], the haze removing from an image, it is important to appraise the transmission map and the global atmospheric light in the image. The creators have proposed the estimation system in light of the base and greatest respective channels. However, the computational expense of the channels is high. The reason for this study is to quicken the rate of the proposed system by utilizing general-purpose computing on graphics processing units (GPGPU) going for the proposed strategy to be utilized as a part of the viable applications. Through the tests, it is affirmed that the count pace of the technique is radically quickened enough for pragmatic use.

Apurva Kumari et.al [9], this paper, a novel and straightforward rebuilding-based fog removal methodology are proposed. Here, we proposed a methodology that depends on the gamma transformation method and median filter. The transmission guide is refined by the gamma transformation method. At that point, the acquired transmission guide is smoothed by a median filter. Subjective and quantitative investigation exhibit that the proposed algorithm performs well in examination with bilateral filtering. It can deal with shading and in addition dark images. The proposed algorithm, because of its pace and capacity to improve visibility, may be used in many systems, ranging from tracking and navigation, surveillance, consumer electronics, intelligent vehicles to remote detecting. The methodology for making the proposed algorithm autonomous of these estimations is to use conceivable improvement systems which build the brilliance of the images. The outcomes are analyzed because of the above-mentioned performance metrics.

Garima Yadav et.al [10], point of this paper is to audit review state-of-art image enhancement and rebuilding techniques for enhancing the quality and perceivability level of an image which give a clear image in terrible climate condition. We likewise think about pervasive methodologies around there through the usage of the systems keeping parameters basic for critical analysis. Many algorithms are developed for improving the visibility quality 
of an image in the spatial domain but if these methods are applied in the frequency domain then they produce a better result and reduce the time to produce the output. When we want to convert the spatial domain input into frequency domain then use Fast Fourier transforms (FFT) and Discrete Fourier transforms (DFT). When we get the frequency of noise then we use filtering methods and reduce the noise frequency and produce is enhanced output improved image.

Di $\mathrm{Wu}$ et. al[11], this paper entirety up the status of image haze removal methods. After that, quantitative and subjective assessments of these strategies are exhibited and the difficulties of the current haze removal method are compressed. At long last, it proposed a few desires for future examination in the field of image haze removal. Compare to the histogram correlation coefficients of the whole image, those of the light haze part are much higher, which means there is a correlation between the histogram of the original image and those of the haze removed images in the light haze area. In other words, the color distortion in the haze removed image is acceptable and not too serious.

F.-C. Chenget. al[12], in this reported work, the lowest level channel former is proposed for image fog removal. The utilization of the most reduced level channel is disentangled from the dark channel prior. It depends on a key perception that fog-free force in a shading image is normally the base estimation of trichromatic channels. To gauge the transmission model, the dull channel former then executes as a min channel for the most reduced force. However, the min channel results in radiance curios, particularly for neighbors of edge pixels. It recalled the transmission model to defog an image; we then accelerated the refinement of transmission by introducing a fast $\mathrm{O}(1)$ bilateral filter based on the raised cosines function to the weight values of neighbors.

\section{Conclusion}

After studying the list of the researcher on fog removal techniques, we come to know that researchers have ignored various issues like no technique works with every phase or circumstance. The existing techniques have neglected the use of dark channels before reducing the noise and uneven illumination problem. To overcome the problems of existing research a new integrated algorithm will be proposed in near future. The trilateral filtering will be used as a post-processing step to eliminate the noise from the input image.

\section{References}

[1] C. Dhanani and A. Saxena, "A Comparative analysis on various Image Enhancement Techniques for Foggy images", International Journal of Advanced Research in Computer Science and Management Studies, vol. 2, no. 12, December (2014)

[2] K. Kumar, G. P. Panda, and N. Panda, "A Survey on Image Dehazing Methods", International Journal of Engineering Research \& Technology (IJERT), vol. 2, no.10, October (2013)

[3] R. Sharma and V. Chopra, "A Review on Different Image Dehazing Methods", International Journal of Computer Engineering and Applications, vol. 6, no. 3, June (2014)

[4] H. Kaur and R. Mahajan, "A Review on Various Visibility Restoration Techniques", International Journal of Advanced Research in Computer and Communication Engineering, vol. 3, no. 5, May (2014)

[5] M.K. Saggud, and S. Singh, "A Review on Various Haze Removal Techniques for Image Processing”, International Journal of Current Engineering and Technology, vol.5, no.3 June (2015)

[6] P. Zhou and Y. Zhou, "Single Image Haze Removal Using Dark Channel Prior and Fields of Experts Model", 11th International Conference on Fuzzy Systems and Knowledge Discovery, IEEE,(2014) 
[7] H. Kawarabuki and K. Onoguchi, "Snowfall Detection in a Foggy Scene", 22nd International Conference on Pattern Recognition, IEEE, (2014)

[8] S. Furukawa, T. Fukuda, T. Koga, N. Suetake and E. Uchino, "High-Speed Min-Max Bilateral Filter-Based ImageDehazing by Using GPGPU”, International Conference on Advanced Mechatronic Systems, IEEE, Kumamoto, Japan, August 10-12, (2014)

[9] A. Kumari, P.J. Thomas and S.K. Sahoo, "Single Image Fog Removal Using Gamma transformation and median filtering", Annual IEEE India Conference (INDICON), (2014)

[10] G. Yadav, S. Maheshwari, and A. Agarwal, "Fog Removal Techniques from Images: AComparative Review and Future Directions", International Conference on Signal Propagation and Computer Technology (ICSPCT), IEEE (2014)

[11] D. Wu1, Q. Zhu, J. Wang, Y. Xie, and L. Wang, "Image Haze Removal: Status, Challenges, and Prospects", IEEE, (2014)

[12] F.-C. Cheng, C.H. Lin, and J.L. Lin," Constant time O(1) image fog removal using lowest level channel", Electronics Letters, vol. 48, no. 22,25th October (2012) 